\title{
ALCC 2021-2022 Quarterly Report
}

Project Title: Informing Forensics Investigations of Nuclear Materials

PI: Ashley Shields

Project ID: CHM161

\section{Project Milestones}

- Please provide the status of all of your milestones/goals from your proposal. Please highlight any project milestones/goals that have changed.

- Structural optimization of mixed defects in graphite: Perform geometry minimization calculations of Dienes $+\mathrm{H}$ and Dienes $+\mathrm{O}$ mixed defects in graphite (target date: August 2021)

- Optimized the structure of 5 Dienes $+O$ substitution defect geometries, 8 Dienes $+\mathrm{O}$ intercalation defect geometries, 5 Dienes $+\mathrm{H}$ substitution defect geometries, and 8 Dienes $+\mathrm{H}$ intercalation defect geometries

- Status: Complete

- Structural optimization of nonstoichiometric $\mathrm{U}_{3} \mathrm{O}_{8}$ : Perform geometry minimization calculations of $\mathrm{U}_{3} \mathrm{O}_{8 \pm z}$ (target date: August 2021)

- Structures for $\mathrm{U}_{3} \mathrm{O}_{7.93}$ and $\mathrm{U}_{3} \mathrm{O}_{7.87}$ (corresponding to the removal of one and two oxygen atoms from the $2 \times 2 \times 2$ supercell) have been generated. There are five inequivalent $\mathrm{U}_{3} \mathrm{O}_{7.93}$ structures and 229 inequivalent $\mathrm{U}_{3} \mathrm{O}_{7.87}$ structures. Relaxations of the $\mathrm{U}_{3} \mathrm{O}_{7.93}$ have begun

- Status: Ongoing

- Structural optimization of defects in $\mathrm{UO}_{\mathrm{x}}$ : Perform geometry minimization calculations of elemental defects in $\mathrm{UO}_{2}, \mathrm{U}_{3} \mathrm{O}_{8}$, and studtite $\left(\mathrm{UO}_{2} \mathrm{O}_{2} \cdot 4 \mathrm{H}_{2} \mathrm{O}\right)$ (target date: August 2021)

- Calculations delayed until early Q2 because of programmatic reasons (reworking final list of elemental defects to target in new fiscal year)

- Status: Waiting on project decisions

- Phonon calculations of mixed defects in graphite: Use the finite displacement method to calculate lattice dynamics for Dienes $+\mathrm{H}$ and Dienes + O mixed defects in graphite (target date: September 2021)

- Phonon calculation inputs have been prepared, and phonon calculations are running

- Status: ongoing 
- Phonon calculations of nonstoichiometric $\mathrm{U}_{3} \mathrm{O}_{8}$ : Use the finite displacement method to calculate lattice dynamics of $\mathrm{U}_{3} \mathrm{O}_{8 \pm z}$ (target date: September 2021)

- Needs completion of corresponding optimizations

- Status: Waiting on dependencies

- Phonon calculations of defects in $\mathrm{UO}_{\mathrm{x}}$ : Use the finite displacement method to calculate lattice dynamics of defects in $\mathrm{UO}_{2}, \mathrm{U}_{3} \mathrm{O}_{8}$, and studtite (target date: September 2021)

- Needs completion of corresponding optimizations

- Status: Waiting on dependencies

- AIMD simulations of perturbed defective graphite: Perform AIMD simulations of $\mathrm{H}, \mathrm{O}$, and vacancy defects in graphite at $300 \mathrm{~K}$ (target date: December 2021)

- Before performing AIMD simulations, we are performing DFT optimization and phonon calculations for static perturbations to downselect perturbations of interest for AIMD

- Status: Preparations are underway

- Amm2 to $P-62 m$ phase transition of $\mathrm{U}_{3} \mathrm{O}_{8}$ : Use geometry minimization, nudged elastic band, and finite displacement method to calculate the solidsolid phase transition and determine spectral activity (target date: December 2021)

- Status: Q2 task, not yet started

- Submit manuscript(s) about $\mathrm{UO}_{\mathbf{x}}$ defects: Submission of manuscript(s) containing results of $\cup_{x}$ defect calculations (target date: December 2021)

- Needs completion of corresponding calculations.

- Status: Waiting on dependencies, Q2 task

- Submit manuscript about mixed defects in graphite: Submission of manuscripts containing results of mixed defects in graphite calculations (target date: January 2022)

- Needs completion of corresponding calculations

- Status: Waiting on dependencies, Q3 task

- H diffusion in graphite: Use geometry minimization, nudged elastic band, and finite displacement method to calculate the potential energy surface of isolated $\mathrm{H}$ diffusion and $\mathrm{H}$ diffusion near a second intercalated hydrogen atom in graphite (target date: March 2022)

- Status: Q3 task, not yet started

- Submit manuscript(s) about $\mathrm{U}_{3} \mathrm{O}_{8}$ : Submission of manuscript(s) containing results of nonstoichiometric and solid-solid phase transition calculations of $\mathrm{U}_{3} \mathrm{O}_{8}$ (target date: March 2022)

- Needs completion of corresponding calculations 
- Status: Waiting on dependencies, Q3 task

- Diffusion barriers of defects in $\mathbf{U O}_{\mathbf{x}}$ (target date: March 2022)

- Needs completion of corresponding calculations to downselect elemental defects

- Status: Waiting on dependencies, Q3/4 task

- Presentation of results at national professional meetings: Present current findings at national conferences hosted by professional societies such as the American Chemical Society or the American Physical Society (target date: March/April 2022)

- Status: Q3/Q4 task, not yet started

- O diffusion in graphite: Use geometry minimization, nudged elastic band, and finite displacement method to calculate the potential energy surface of isolated $\mathrm{O}$ diffusion and $\mathrm{O}$ diffusion near a second intercalated oxygen atom in graphite (target date: June 2022)

- Status: Q4 task, not yet started

- Submit manuscript(s) about diffusion in $\mathrm{UO}_{\mathrm{x}}$ : Submission of manuscript(s) containing results of diffusion barrier calculations (target date: June 2022)

- Needs completion of corresponding calculations

- Status: Waiting on dependencies, Q4 task

- Submit manuscripts about temperature dependent lattice dynamics and defect diffusion in graphite: Submission of manuscripts containing results of AIMD simulations at elevated temperature and defect diffusion pathways in graphite (target date: August 2022)

- Needs completion of corresponding calculations

- Status: Waiting on dependencies, Q4 task

- If not covered above, list major accomplishments thus far for your ALCC Project. Please include scientific and computational details of simulations undertaken, including images if possible.

All accomplishments thus far have been covered above.

- Describe how access to leadership computing resources, data capabilities, and/or staff at the Leadership Computing Facility (LCF) enabled these results.

Simulating experimental observables, particularly vibrational spectroscopic features, requires the calculation of phonons. Additionally, calculating the phonons of defective materials requires large supercells to minimize interactions with periodic images and a large number of calculations in the finite displacement method owing to the low translational symmetry of the material. These numerous, large-scale calculations are only feasible on leadership computing resources. 


\section{Project Productivity}

Please remember that all publications, which involve the use of ALCC resources, should contain the following acknowledgement: This research used resources of the Oak Ridge Leadership Computing Facility, which is a DOE Office of Science User Facility supported under Contract DE-AC05-00OR22725.

- List and provide links if possible to publications that resulted from the use of your ALCC project.

1. Isbill, S. B., A. E. Shields, R. J. Kapsimalis, J. L. Niedziela, "Computational investigations of Dienes defect-and vacancy-induced changes to the electronic and vibrational properties of carbon fiber structural units." Work executed under ALCC CHM161 2020-2021 has been submitted to Phys. Chem. Chem. Phys. for review.

- List invited talks or presentations.

Currently no invited talks or presentations associated with this ALCC.

- List any journal covers, awards, honors, and/or popularizations.

Currently no journal covers, awards, honors, and/or popularizations associated with this ALCC.

- List any formal software releases generated as a result of this project.

Currently no formal software releases associated with this ALCC.

- Technical Accomplishments_List technical accomplishments such as development of reusable code resulting in a new tool, new algorithm design ideas or programming methodologies, formal software releases, etc.

While we adapt our own postprocessing and analysis codes as needed, these will not be publicly released.

- List any patents (filed or received).

Currently no patents associated with this ALCC.

- List any invention disclosures.

Currently no invention disclosures associated with this ALCC. 
- Other

\section{Center Feedback}

- Have the LCF systems met the needs of your project (e.g., queuing policies, etc.)?

Yes, the LCF systems have met our project needs so far. Wall times and the queueing policy are very reasonable.

- If utilized, has the support received from the User Assistance been beneficial to your project team?

We reached out to the User Assistance team to gain access to a module that runs a proprietary software. The User Assistance team quickly verified we had an active license for that software and gave us access within a few hours, allowing us to quickly get calculations using this software running. A new VASP module was built very quickly after the system upgrade and shared with the user community.

- Are you aware of the monthly user telecons? If so, do you participate and find them useful? How could we improve the telecons?

Yes, we are aware of the monthly user telecons. We occasionally participate when topics are relevant to our work, which does not involve heavy programming or software development.

- Is the website (www.olcf.ornl.gov) meeting your needs? What areas can be improved to better assist you?

Yes, the website is meeting our needs. Topics in the user documentation are generally easy to find and the new myOLCF is very helpful in monitoring our allocation usage.

- Are there any particular training classes that you and your project team would like to see the OLCF offer in the future?

I'm not currently aware of any training classes that our team would like to see offered.

- Any suggestions for improvement/areas where we can assist you that have not been discussed?

I currently have no suggestions on improvements. Thanks for your great work!

\section{Outreach}


The center creates (concise, short, highly visible) bi-weekly center highlights to submit to DOE. Please let us know if your project is ready, willing, and able to contribute a highlight?

Our project is not currently ready to contribute to a highlight.

\section{Code Description and Characterization}

- Name and provide a description of the primary codes used by your project.

Real-space MultiGrid (RMG): Density functional theory software for periodic systems which utilizes real-space integration for improved parallelization. Built and running on Summit.

Vienna ab initio Simulation Package (VASP): Commonly used density functional theory software for periodic systems that offers reliable modeling of actinide oxides that are known to have complex electronic structures. Running on Summit using VASP module.

Phonopy: Postprocessing software for the calculation of phonons from standard density functional theory software, as well as a variety of analysis tools. Built and running on Andes.

- What languages and libraries (scientific, $\mathrm{I} / \mathrm{O}$, etc.) are used in each code?

- If possible and useful, please indicate which of the following algorithmic motifs appear in each of your major production codes.

\begin{tabular}{|l|l|l|l|l|l|l|l|l|}
\hline $\begin{array}{l}\text { Code } \\
\text { Name }\end{array}$ & $\begin{array}{l}\text { Dense } \\
\text { Linear } \\
\text { Algebra }\end{array}$ & $\begin{array}{l}\text { Sparse } \\
\text { Linear } \\
\text { Algebra }\end{array}$ & $\begin{array}{l}\text { Monte } \\
\text { Carlo }\end{array}$ & FFTs & Particles & $\begin{array}{l}\text { Structured } \\
\text { Grids }\end{array}$ & $\begin{array}{l}\text { Unstructured } \\
\text { Grids }\end{array}$ & AMR \\
\hline & & & & & & & & \\
\hline & & & & & & & & \\
\hline
\end{tabular}

